\title{
Effectiveness and safety of dual antiplatelet therapy in coronary aneurysms caused by Kawasaki disease in children: study protocol for a multicenter randomized clinical trial
}

\author{
Dan $\mathrm{Li}^{1 \#}$, Xi Chen ${ }^{2 \#}$, Xiaohui Li $^{1}$, Yue Yuan ${ }^{2}$, Hongfang Jin ${ }^{3}$, Guiying Liu ${ }^{4}$, Huili Zhang ${ }^{5}$ Gaoqiang Xie ${ }^{6}$ \\ ${ }^{1}$ Department of Cardiology, Children's Hospital Capital Institute of Pediatrics, Graduate School of Peking Union Medical College, Beijing, China; \\ ${ }^{2}$ Department of Cardiology, Beijing Children's Hospital, Capital Medical University, Beijing, China; ${ }^{3}$ Department of Pediatrics, Peking University \\ First Hospital, Beijing, China; ${ }^{4}$ Department of Pediatrics, Beijing Anzhen Hospital, Capital Medical University, Beijing, China; ${ }^{5}$ Department of \\ Cardiac Surgery, Fuwai Hospital, National Center for Cardiovascular Disease, Chinese Academy of Medical Sciences and Peking Union Medical \\ College, Beijing, China; ${ }^{6}$ Clinical Research Institute of Peking University, Beijing, China \\ Contributions: (I) Conception and design: D Li, X Chen, X Li, Y Yuan; (II) Administrative support: X Li, Y Yuan; (III) Provision of study materials or \\ patients: X Li, Y Yuan, H Jin, G Liu, H Zhang; (IV) Collection and assembly of data: D Li, X Chen; (V) Data analysis and interpretation: D Li, X Li; \\ (VI) Manuscript writing: All authors; (VII) Final approval of manuscript: All authors. \\ \#These authors contributed equally to this work. \\ Correspondence to: Xiaohui Li, MD, PhD. Children's Hospital Capital Institute of Pediatrics, Graduate School of Peking Union Medical College, No.2 \\ Ya-Bao Road, Chaoyang District, Beijing 100020, China. Email: lxhmaggie@126.com; Yue Yuan, MD. Department of Cardiology, Beijing Children’s \\ Hospital, Capital Medical University, No.56 Nanlishi Road, Xicheng District, Beijing 100045, China. Email: Yuanyuebj22@sina.com.
}

Background: Medium or large coronary artery aneurysm (CAA) is a serious complication of Kawasaki disease $(\mathrm{KD})$ and may cause thrombosis, coronary artery stenosis, and even myocardial infarction at different stages of the disease. Dual antiplatelet therapy (aspirin plus clopidogrel) is considered for prophylaxis of coronary thrombosis in KD presenting with more than medium CAAs based on adult coronary artery disease experience and expert consensus. This paper describes the study protocol for a randomized controlled trial that measures the clinical effectiveness and safety of dual antiplatelet therapy in the thromboprophylactic treatment of KD.

Methods: The present study is a multicenter, open-label, randomized controlled trial (RCT) with a 12-month follow-up. KD patients with medium or large CAAs (Z-value $\geq 5$ ), evaluated by echocardiogram and clinicians before treatment, are randomized in a 1:1 ratio and assigned to aspirin therapy alone (Control group), or dual antiplatelet (aspirin plus clopidogrel) therapy (Experimental group). Antiplatelet therapy is given to the KD patients from the time of diagnosis until the coronary artery returns to normal. Weekly or monthly follow-up visits are conducted to record compliance, recovery, and biochemical indicators and continue for one year. The primary outcome is the incidence of thrombus throughout the disease and the effective of dual antiplatelet. The secondary outcomes are the safety of dual antiplatelet drugs, plateletrelated indicators, inflammatory indicators, biochemical indexes and drug-related indicators during the study period. Patients who do not meet the inclusion criteria of the RCT trial or those unwilling to provide informed consent enter the registration trial.

Discussion: This is the first study to evaluate the effectiveness and safety of dual antiplatelet therapy in coronary aneurysms caused by Kawasaki disease in children. It is hoped that this study will play an important and significant role in improving the prognosis and long-term quality of life for children with KD complicated by CAAs.

Trial registration: Chinese Clinical Trial Registry, ChiCTR1800019181. Registered on Oct 30, 2018.

Keywords: Kawasaki disease; aspirin; clopidogrel; coronary artery aneurysm 
Submitted Feb 24, 2021. Accepted for publication May 21, 2021.

doi: $10.21037 / \mathrm{tp}-21-74$

View this article at: http://dx.doi.org/10.21037/tp-21-74

\section{Introduction}

Kawasaki disease (KD) is a self-limited vasculitis of unknown etiology that is the most common cause of acquired heart disease in children in developed countries (1), and which preferentially affects medium-sized arteries, particularly the coronary arteries (CA) (2). According to the criteria of the American Heart Association (AHA), 20\% of KD patients will develop transient coronary artery dilation, $5 \%$ will develop coronary artery aneurysms (CAA) ( $\mathrm{Z}$-value $>2.5$ ), and approximately $1 \%$ will develop giant CAAs (3). Patients with large or giant CAAs are at high risk for cardiac events, including CA thrombosis, stenosis, myocardial infarction, and even sudden cardiac death (4). Antiplatelet agents are used for the prophylaxis of coronary thrombosis in KD. For patients with small CAAs, monotherapy with lowdose aspirin is sufficient for the prophylaxis of thrombosis. However, for patients with medium, large, or giant CAAs, dual antiplatelet therapy, such as aspirin combined with a thienopyridine (e.g., clopidogrel), has been recommended as prophylaxis against coronary artery thrombosis in the AHA scientific statement (3).

The AHA scientific statement regarding antiplatelet therapy in KD with medium or giant CAAs relies on reasoning from retrospective studies, practices in atherosclerotic coronary artery disease, and expert consensus (3). To date, there have been no randomized clinical trials evaluating the effectiveness and safety of antithrombotic regimens for the prophylaxis of coronary thrombosis in children with KD. This study aims to investigate the effectiveness and safety of dual antiplatelet therapy as thromboprophylaxis for KD patients with medium or giant CAAs from the time of diagnosis to the day of discharge, and then at 2 weeks, 1, 2, 3, 6, and 9 months, and 1-year after discharge from hospital. We hypothesize that dual antiplatelet therapy will reduce the formation of thrombosis, improve the recovery of the CAs, change the $Z$ scores, and reduce the occurrence of adverse events (AEs). We present the following article in accordance with the SPIRIT reporting checklist (available at http:// dx.doi.org/10.21037/tp-21-74).

\section{Primary objective}

(I) To measure the effectiveness of dual antiplatelet therapy for coronary aneurysms caused by KD in children with medium or giant CAAs;

(II) To measure whether the dual antiplatelet therapy (aspirin plus clopidogrel) could improve the anticoagulation function of KD patients in the index of prothrombin time (PT), D-Dimer and international normalized ratio (INR).

\section{Secondary objectives}

(I) To measure the safety (treatment-related AEs) of dual antiplatelet drugs;

(II) To measure the $Z$-scores and compare the change in $Z$-scores of the two groups at different time points during the 1-year follow-up;

(III) To measure whether dual antiplatelet therapy in the acute and subacute phase of KD reduces the levels of inflammatory markers, such as $\mathrm{C}$ reactive protein (CRP), white blood cell count (WBC), and circulating neutrophil count (NEUT) in KD patients with medium or giant CAAs;

(IV) To measure whether the treatment of aspirin plus clopidogrel has an influence on biochemical indexes, such as alanine aminotransferase (ALT) and aspartate aminotransferase (AST) in KD patients in the RCT;

(V) To measure drug-related indicators, such as the inhibitory rate of platelet aggregation, 11-dehydrothromboxane, CYP2C19, and the cyclooxygenase gene at baseline (pre-clopidogrel at diagnosis), discharge, and 1-year follow-up;

(VI) To register the information of the KD patients and measure the risk factors leading to coronary artery thrombosis.

\section{Study design}

In our cohort study, we have included an RCT and registration study. The RCT is a 1-year, two-arm, 


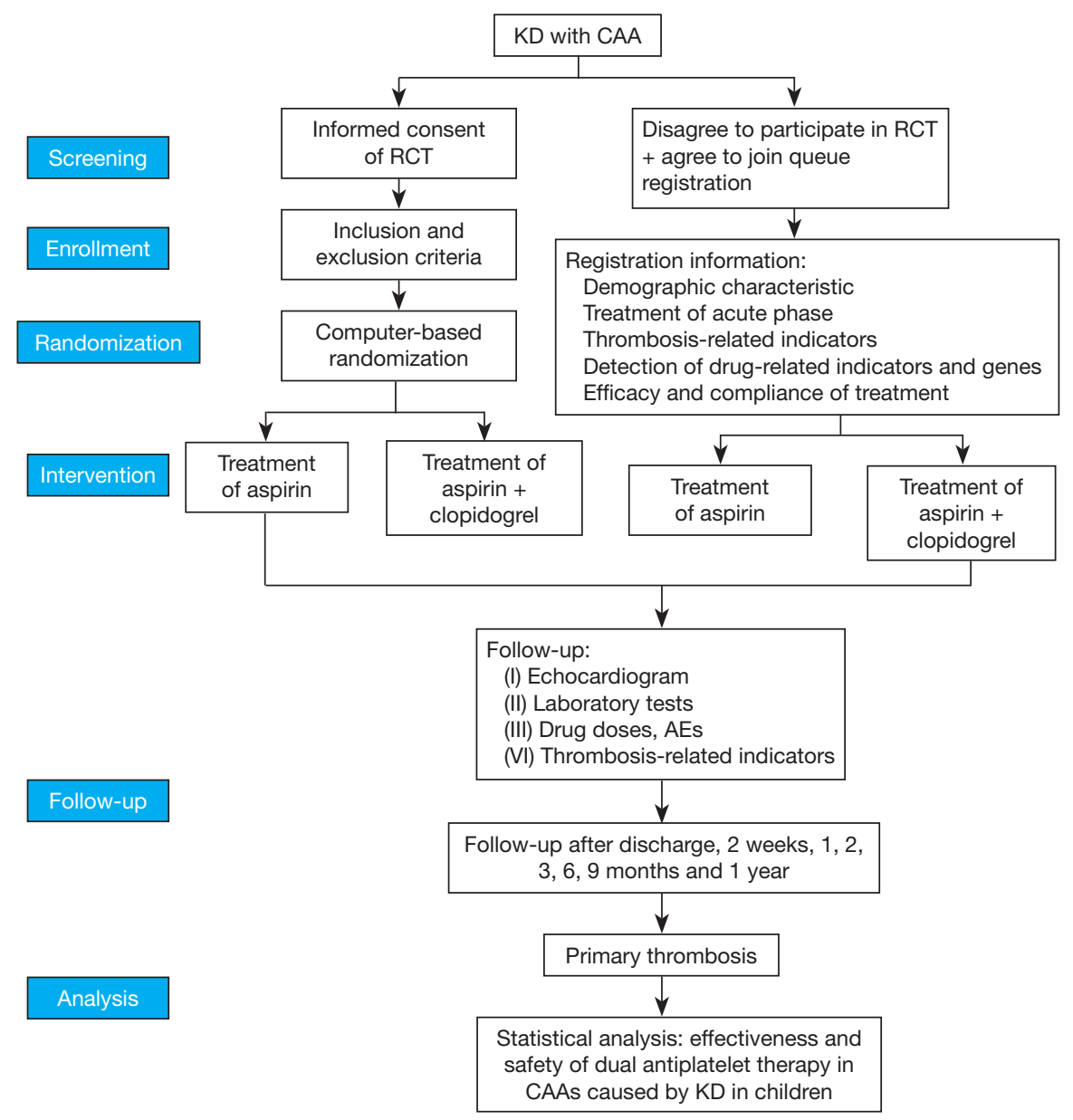

Figure 1 KDDATE study design.

randomized, multicenter study comparing aspirin plus clopidogrel with aspirin alone for prophylactic treatment of CA thrombosis in children with KD. Eligible patients are required to sign the informed consent form (no. SHERLL2018020) and are randomly assigned to either Group A (aspirin alone) or Group B (aspirin plus clopidogrel) by the REDCap randomization system. The registration trial registers patient information, including demographic data, clinical symptoms, the treatment regimens in the acute phase of the disease, and results of thrombosis-related laboratory tests.

\section{Methods}

\section{Participating hospitals}

Patients are recruited mainly from five pediatric and cardiology hospitals in Beijing. The principal investigators at the participating hospitals are pediatricians and cardiologists with at least 5 years of experience in pediatric cardiology treatment and who specialize in pediatric cardiology education.

Study participants who develop complications, AEs, coronary artery aggravations, or who drop out of the follow-up phase of the study are deemed "therapeutic failures". Children whose parents or legal guardians are unable or unwilling to join the RCT cohort are added to the registration cohort (Figure 1). Figure 1 shows the study flow chart.

\section{Patient involvement}

The members of our study team determined the inclusion and exclusion criteria of the trial. The parents or guardians of the participants are fully informed and are required to 
Table 1 Published definitions of coronary artery abnormalities assessed by echocardiography of the AHA (3)

$\begin{array}{ll}\text { Small aneurysm } & \begin{array}{l}\text { Aneurysms with internal luminal diameter } \leq 4 \mathrm{~mm} \text {. In children } \geq 5 \text { years, internal luminal diameter of a segment } \\ \text { measures }>1.5 \text { times that of an adjacent segment; or } 2.5 \leq Z \text { value }<5\end{array} \\ \text { Medium aneurysm } & \begin{array}{l}\text { Aneurysms with an internal luminal diameter of }>4 \mathrm{~mm} \text { to } \leq 8 \mathrm{~mm} \text {. In children } \geq 5 \text { years, the internal luminal diameter } \\ \text { of a segment measures } 1.5 \text { to } 4 \text { times that of an adjacent segment; or } 5 \leq Z \text { value }<10\end{array}\end{array}$

Giant aneurysm Aneurysms with an internal luminal diameter of $>8 \mathrm{~mm}$. In children $\geq 5$ years, the internal luminal diameter of a segment measures $>4$ times that of an adjacent segment; or $Z$ value $\geq 10$

Z-value is the size of the internal luminal diameter adjusted by body surface area.

sign informed consent. Patients who meet the inclusion criteria are enrolled and then randomly assigned to either group A (aspirin alone) or group B (aspirin plus clopidogrel) by the REDCap randomization system. All patients who are given antithrombotic therapy enter the follow-up cohort. The antithrombotic therapy is evaluated by the expert cardiology members of the study team at discharge, and then at 2 weeks, 1, 2, 3, 6, and 9 months, and 1-year post-discharge. Coronary computed tomography (CT) or coronary angiography will be made when the thrombosis is suspected or in the six-month during the course of the disease.

\section{Inclusion criteria of the RCT trial}

Inclusion criteria are as follows: (I) 4 weeks to 18 years of age; (II) patients diagnosed with KD who meet five of the six clinical KD criteria (febrile days $\geq 5$; rash; erythema and cracking of lips and strawberry tongue; bilateral bulbar conjunctival injection without exudate; erythema and edema of the extremities in the acute phase and/or periungual desquamation in the subacute phase; and cervical lymphadenopathy); (III) patients diagnosed with incomplete $\mathrm{KD}$ who meet two or three of the clinical KD criteria besides fever accompanied by CAA as shown by twodimensional echocardiography or coronary angiography; (IV) patients with KD hospitalized for the first time; (V) patients with medium or large CAAs; (VI) KD patients with small CAAs at diagnosis which extend to medium or large CAAs within one month of disease onset.

\section{Exclusion criteria of the RCT trial}

Exclusion criteria are as follows: (I) patients with a history of hypersensitivity to aspirin or clopidogrel; (II) patients with gastrointestinal ulcers; (III) patients demonstrating abnormal liver enzymes; (IV) patients with hemorrhagic disease; (V) KD concomitant with other vasculitis diseases. All CA assessments are performed by two-dimensional echocardiography (Table 1).

\section{Randomization, allocation, and blinding}

Eligible patients are randomly assigned to either Group A (aspirin alone) or Group B (aspirin plus clopidogrel) by the REDCap randomization system. In the RCT cohort, a randomization scheme is used to ensure balanced allocation of subjects to the study arms, such that 40 subjects are assigned to each treatment group (allocation ratio 1:1) and equal numbers are assigned to receive aspirin or aspirin plus clopidogrel (Figure 1). Allocation is concealed and stratified by the site $(\mathrm{n}=5)$, age, and gender. Records of individual assignments to RCT Group A (aspirin alone) or Group B (aspirin plus clopidogrel) are de-identified (user no. and hospital ID instead of patients' names), sequentially numbered, and stored on a secure computer system until the completion of the program (Figure 2).

\section{Intervention}

\section{Dual antiplatelet therapy and the control group}

After eligible participants sign the informed consent form (no. SHERLL2018020), they are randomly assigned to either Group A (aspirin alone) or Group B (aspirin plus clopidogrel) by the REDCap randomization system. Aspirin is given at $3-5 \mathrm{mg} / \mathrm{kg}$ once a day, and clopidogrel is given at $0.2-3 \mathrm{mg} / \mathrm{kg}$ once a day, depending on the different CYP2C19 genotype. The patients' CYP2C19 genotype determines the clopidogrel dose given to the $\mathrm{KD}$ patients. CYP2C19 extensive metabolizers and ultra-rapid metabolizers are given $0.2-1 \mathrm{mg} / \mathrm{kg} / \mathrm{d}$, and intermediate metabolizers and poor metabolizers are given $1-2 \mathrm{mg} / \mathrm{kg} / \mathrm{d}$, or even $2-3 \mathrm{mg} / \mathrm{kg} / \mathrm{d}$, depending on the result of the inhibitory rate of blood platelet aggregation.

\section{Data entry and data export}

Data collection is performed using a locally developed software called REDCap. The data is collected during hospitalization, after discharge, and at 1-year follow-up. The REDCap dataset is an informatics tool that improves 


\begin{tabular}{|c|c|c|c|c|c|c|c|c|}
\hline \multirow[b]{3}{*}{ TIMEPOINT** } & \multicolumn{8}{|c|}{ Study Period } \\
\hline & \multirow{2}{*}{$\begin{array}{c}\text { Enrolment } \\
\text { Admission }\end{array}$} & \multirow{2}{*}{\begin{tabular}{c|} 
Enrolment \\
0
\end{tabular}} & \multicolumn{5}{|c|}{ Post-allocation } & \multirow{2}{*}{\begin{tabular}{|c|} 
Close-out \\
$t_{x}$
\end{tabular}} \\
\hline & & & Discharge & $30 \mathrm{~d}$ & $60 \mathrm{~d}$ & $3 \mathrm{mo}$ & etc. & \\
\hline \multicolumn{9}{|l|}{ ENROLMENT: } \\
\hline \multirow{4}{*}{$\begin{array}{l}\text { Eligibility screen } \\
\text { Informed consent } \\
\text { Inclusion and } \\
\text { exclusion criteria }\end{array}$} & $x$ & & & & & & & \\
\hline & $x$ & & & & & & & \\
\hline & $x$ & & & & & & & \\
\hline & & $x$ & & & & & & \\
\hline \multicolumn{9}{|l|}{ INTERVENTIONS: } \\
\hline \multicolumn{9}{|l|}{ Aspirin } \\
\hline \multicolumn{9}{|l|}{ Clopidogrel } \\
\hline \multicolumn{9}{|l|}{ ASSESSMENTS: } \\
\hline $\begin{array}{r}\text { Patients' } \\
\text { demographic data }\end{array}$ & $x$ & $\mathrm{x}$ & & & & & & \\
\hline $\begin{array}{l}\text { laboratory } \\
\text { indicators }\end{array}$ & $x$ & $\mathrm{X}$ & & & & & & \\
\hline $\begin{array}{r}\text { Incidence of } \\
\text { thrombus }\end{array}$ & & & & & & & & $x$ \\
\hline Z-value & $x$ & $x$ & & & & & $\longrightarrow$ & $x$ \\
\hline $\begin{array}{r}\text { Treatment-related } \\
\text { AEs }\end{array}$ & & & & & & & $\longrightarrow$ & $x$ \\
\hline $\begin{array}{r}\begin{array}{r}\text { Platelet-related } \\
\text { indicators }\end{array}\end{array}$ & $x$ & & & & & & $\longrightarrow$ & $x$ \\
\hline $\begin{array}{r}\text { Thrombosis-related } \\
\text { indicators }\end{array}$ & $x$ & & & & & & $\longrightarrow$ & $x$ \\
\hline $\begin{array}{r}\text { Drug-related } \\
\text { indicators }\end{array}$ & & & & & & & $\longrightarrow$ & $x$ \\
\hline
\end{tabular}

Figure 2 Schedule of enrolment, interventions, and assessments*. *, recommended content can be displayed using various schematic formats. See SPIRIT 2013 Explanation and Elaboration for examples from protocols. **, list specific timepoints in this row.

the efficiency and accuracy of data retention and has been developed to randomize the study patients. REDCap has a checking function and set ranges for the relevant indicators. The Data Safety Monitoring Board (DSMB) of REDCap reviews the data sent by the study group every month. The attribution of the relatedness of any AEs to the study drugs will be judged by our Adverse Events Committee.

The Case Report Form (CRF) forms and research manuals are initially constructed and then transferred to the study's electronic database (REDCap). This program is also registered on the Chinese Clinical Trial Registry (no. ChiCTR1800019181).

\section{Follow-up}

We keep track of the enrolled children by a mobile-based outreach team. Enrolled children are visited weekly or monthly during the follow-up period, and our interviewers collect information about their physical condition, treatment compliance (in both study groups), usage of medication, clinical manifestations, and any AEs (Figure 2).

\section{Outcome assessment}

The outcome measurement of CAs and thrombus is primarily evaluated by two-dimensional echocardiography, coronary computed tomography (CT), or coronary angiography during the course of $\mathrm{KD}$. The frequency of $\mathrm{CT}$ is determined according to the children's coronary arteries but is always used in KD patients who continue to have medium or large CAAs at the 1-year follow-up. Additional outcome measures include treatment-related AEs, platelet- 
related indicators, biochemical indexes, thrombosis-related indicators, and biochemical and drug-related indicators. These indicators are evaluated by changes in the laboratory indexes before and after treatment in the groups (Figure 2).

\section{Primary outcome}

The primary outcome measure is the incidence of thrombus throughout the disease from initial admission to 1-year follow-up and the anticoagulation function of the dual antiplatelet therapy in the $\mathrm{KD}$ patients.

\section{Secondary outcomes}

Additional outcomes include the safety of dual antiplatelet drugs (treatment-related AEs), the Z-score and the change of $\mathrm{Z}$-score in the two groups, inflammatory conditions, biochemical indexes, and drug-related indicators at baseline (pre-clopidogrel at diagnosis), discharge, and 1-year follow-up. AEs and drug-related indicators are assessed on admission, discharge, and during the study period (at 2 weeks, 1, 2, 3, 6, and 9 months, and 1-year follow-up). The onset of fever marks the first day of the disease. Parents are asked to observe clinical manifestations and AEs at home after discharge, such as petechiae, bruises, bleeding, or other reactions. The coronary arteries, including the left main coronary artery (LMCA), the left anterior descending (LAD), the left circumflex artery (LCX), and the right coronary artery (RCA), are evaluated by two-dimensional echocardiography and graded by the largest $\mathrm{Z}$-value (the measurement method are decided by the published definitions (Table 1) (3), which is the size of the internal luminal diameter adjusted by body surface area. The $Z$-value calculation system of Kobayashi (http://raise.umin.jp/zsp/ calculator/) is used in the present study.

The patients who do not meet the inclusion criteria of the RCT or those unwilling to provide informed consent are entered into the registration trial. The outcome is to investigate the patients' demographic data (such as gender, age, and weight), medical history, laboratory indicators (WBC, NEUT, HGB, CRP, ALT, AST, PLT, WPV, MPV, Fib, PT, APTT, PAGT, 11-dehydrothromboxane, CYP2C19, and cyclooxygenase gene) and regimens in the acute phase of the disease [such as intravenous immunoglobulin (IVIG), aspirin, clopidogrel, dipyridamole, low molecular weight heparin, and warfarin], to analyze the risk factors of CAs and thrombosis in $\mathrm{KD}$ patients with medium or large CAAs throughout the 1-year follow-up.

\section{Safety measurements and AE reporting}

Any unexpected AEs occurring during the intervention and follow-up period are reported to the research assistants, and the causality of drugs and effectiveness is assessed. If serious AEs occur, the research assistants report them to the project manager and ethics committee immediately, deciding whether the participant needs to withdraw from the study. All AEs are followed up until satisfactory resolution or until the treating physicians and the principal investigator deem the event to be chronic or the participant to be stable.

\section{Data collection}

Research assistants collect the demographic characteristics at the time of recruitment. The primary and secondary outcome data are collected by the outcome assessors at baseline, after discharge, and during the follow-up period. All data collected in this study is performed using standardized procedures, and all outcome assessors receive standard training on how to measure outcomes to ensure equal testing conditions for all participants. We are also collecting, evaluating, and storing biological specimens for genetic and molecular analysis in the current trial and for future use in ancillary studies.

\section{Database cooperation center}

In addition to working with five hospitals, we have also cooperated with the Clinical Research Institute of Peking University to construct the computer data system (REDCap), which helps us record and verify the authenticity and validity of the data. It also enables us to check any omissions, correct errors, and conduct statistical analyses.

\section{Power and sample size}

In the RCT cohort, according to our preliminary experimental results, for the Kawasaki disease patients with medium or large coronary artery aneurysm, 14 children were treated with aspirin and 6 of them developed thrombosis, 18 children were treated with aspirin plus clopidogrel and 3 of them developed thrombosis, the incidence of thrombosis in group A (aspirin) was $42.6 \%$ $\left(\mathrm{P}_{\mathrm{T}}=42.6 \%\right)$, and in group B (aspirin plus clopidogrel) was 
16.7\% $\left(\mathrm{P}_{\mathrm{C}}=16.7 \%\right)$. Because the limited cases met the study conditions, the significance level was deemed unilateral, and a statistically significant difference was set at $\alpha=0.05$, $\beta=0.08$. The follow-up drop-out rate was $5 \%$, owing to good parental compliance. We use the sample size formula for calculating the sample size to calculate the sample size and a total number of 80 cases, with 40 cases per group.

\section{Time frame}

This study is designed to conclude in 12 months, including enrollment, intervention, post-discharge follow-up for effectiveness and safety assessments, and data analysis. All patients receive antithrombotic therapy and enter the followup cohort after discharge. The effectiveness and safety of the antiplatelet treatment are evaluated at 2 weeks, 1, 2, 3, 6, and 9 months and 1-year post-discharge. If any enrolled children have medium or large coronary aneurysms after 1 year of observation, the follow-up period will be extended.

\section{Statistical analysis plan}

This is a randomized controlled cohort study. Results will be reported as point estimates (risk ratios, odds ratios, or mean differences, as appropriate) and interval estimates ( $95 \%$ confidence intervals). All tests of significance for the secondary outcomes will be 2-sided, and Holm's adjustments will be made for multiple comparisons. We will use the $\chi^{2}$ test or Fisher's exact test for categorical variables. Summary statistics (means and SD) of patients' characteristics will be calculated, and a two-sample $t$-test or Mann-Whitney $U$ test will be used for continuous variables to compare the demographic and baseline characteristics between the two groups. Time to all-cause discontinuation will be summarized by using Kaplan-Meier estimates and compared with the log-rank test. All $\mathrm{P}$ values are 2-tailed, and the statistical significance is set at $\mathrm{P}=0.05$. Statistical analysis will be conducted using the statistical software $\mathrm{R}$ 4.0.2. (www.rproject.org). The Data and Safety Monitoring Board (DSMB) of the Clinical Research Institute of Peking University designed our computer data system (REDCap) plans to conduct interim and final analyses and modify for efficacy or deficiency during ongoing safety monitoring conducted for this study.

\section{Ethics and informed consent (SHERLL2018020)}

The study will be conducted in accordance with the
Declaration of Helsinki (as revised in 2013). The study protocol was reviewed and approved by the institutional review board of all participating centers. Six centers are participating in this program, all of which have signed the cooperation agreement. Written informed consent from the parents or legal guardians will be obtained for all participants.

\section{Publication policy}

The results of the trial will be published in peer-reviewed journals and presented at relevant congresses. The trial will be reported following the CONSORT recommendations.

\section{Discussion}

KD was first proposed by a Japanese physician in 1967 and is an acute self-limited vasculitis that primarily affects infants and young children (3). CAAs rated as more than medium are serious complications of $\mathrm{KD}$, placing patients at risk for coronary thrombosis or stenosis, myocardial infarction, and sudden cardiac death (5). Studies on CA stenosis and occlusion caused by KD complicated with CAAs have found that the size of CAAs and the number of coronary arteries involved are the main factors for thrombosis (6). Tsuda et al. reported that a CA with an internal lumen diameter exceeding $4 \mathrm{~mm}$ would lead to intima thickening during the recovery period(7). Bang et al. reported that the incidence of CA stenosis or occlusion was $42.3 \%$ with an internal lumen diameter exceeding $6 \mathrm{~mm}(8)$, and Suda et al. found that the incidence of CA stenosis or complete occlusion was caused by giant CAA was $61 \%$ (9).

2017 AHA scientific statement (3) of KD mentioned that KD arteriopathy included 3 pathological processes of which the second process is a subacute/chronic vasculitis characterized by an asynchronous infiltration of lymphocytes, plasma cells, and eosinophils with fewer macrophages that begins in the first 2 weeks after fever onset but can continue for months to years in a small subset of patients. Based on this pathological finding, we chose clopidogrel improving inflammatory conditions of $\mathrm{KD}$ patients as the objective in order to take advantage of the its dual effects with anti-platelet and anti-inflammatory.

The use of antiplatelet drugs for the primary and secondary prevention of cardiovascular and cerebrovascular thromboses in adult populations has been evaluated, resulting in defined management guidelines (10). Much less is known about the use of antiplatelet drugs to prevent 
thrombosis in infants and children (11). Prior studies have found that the incidence of CA stenosis or occlusion was $46-61 \%$ in Japan (11), $42.3 \%$ in South Korea (10), and $34.6 \%$ in America (12) with low-dose aspirin antiplatelet therapy alone, which suggests that aspirin alone is not enough to prevent CA thrombosis. Aspirin may not be sufficient to prevent thrombosis because it inhibits only the cyclooxygenase pathway, leaving other platelet activation pathways unaffected (13). Clopidogrel, a thienopyridine derivative, produces its antiplatelet effect through an active metabolite that specifically and irreversibly modifies the adenosine diphosphate (ADP) purinergic $\mathrm{P} 2 \mathrm{Y}_{12}$ platelet receptor and through the subsequent $\mathrm{ADP}$-mediated activation of the glycoprotein IIb/IIIa complex that inhibits platelet aggregation (14). Clopidogrel is a potent oral antiplatelet agent used in conjunction with aspirin to treat CA disease, peripheral vascular disease, and cerebrovascular disease in adults (15). However, there are few published studies on the use of clopidogrel in children, especially on the safety and optimal dosage of clopidogrel in children with $\mathrm{KD}$. Because of the limited patient sample sizes and the small number of thrombotic events, there have been no randomized clinical trials evaluating the safety and effectiveness of antiplatelet regimens for the prophylaxis of CA thrombosis in KD. Our trial of dual antiplatelet therapy in coronary aneurysm caused by Kawasaki disease (KDDATE), which includes an RCT and registration trial, is the first to compare the safety and effectiveness of dual antiplatelet (aspirin plus clopidogrel) therapy for CAA caused by KD in children, and the registration trial may also provide a foundation to determine the risk factors for KD patients with medium or large CAAs. Clopidogrel is a potent oral antiplatelet agent used for the secondary prevention of arterial thrombosis in adult patients (16). Low et al. reviewed KD patients with a medium to large CAAs receiving combination thromboprophylactic therapy (dual or triple therapy with ASA, clopidogrel, and low molecular weight heparin (LMWH) or warfarin), and concluded that the overall bleeding risk was lower in $\mathrm{KD}$ patients receiving combination thromboprophylaxis than single drug (17).

A previous prospective, multicenter, randomized, placebocontrolled trial (4) evaluating the pharmacodynamics of clopidogrel in children aged $0-24$ months of age with a cardiac condition at risk for arterial thrombosis found that clopidogrel $0.20 \mathrm{mg} / \mathrm{kg} / \mathrm{d}$ achieved a platelet inhibition level similar to that of adults taking $75 \mathrm{mg} / \mathrm{d}$. In other words, clopidogrel is well tolerated in infants and young children at this dose, and there were no serious adverse reactions. These results provide the basis for further usage of clopidogrel for antiplatelet therapy in children. The data indicated that the dose of $0.20 \mathrm{mg} / \mathrm{kg}$ achieved the target mean of $30-50 \%$ inhibition of $5 \mu \mathrm{mol} / \mathrm{L}$ ADP-induced platelet aggregation for both the maximum extent and the rate of platelet aggregation (4). Studies have shown that the enzymes encoded by the CYP2C19 gene influence clopidogrel metabolism by affecting the absorption and activation of clopidogrel (18). In the present study, different dosages of clopidogrel are given according to the patient's CYP2C19 genotype to explore the optimal dosage in children with $\mathrm{KD}$. In order to reduce the clinical adverse events after clopidogrel was used, CYP2C19 gene detection has become a common detection method to guide clinical treatment. CYP2C19 genotypes are detected by sequencing method and gene chip generally. Detection of CYP2C19 genotypes can be used to individualize clopidogrel, which is of great significance to reduce cardiovascular mortality.

In a study of patients younger than 40 years of age undergoing cardiac catheterization for suspected ischemia, $5 \%$ of patients had coronary artery lesions consistent with $\mathrm{KD}$ (19). System dynamics modeling suggests that by 2030 , one in every 1,600 adults in the US will have suffered from KD (20). Thus, it is incumbent upon pediatricians to provide the best possible prophylaxis against CAA thrombus, motivating this trial. In this regard, this study plays an important and significant role in improving the prognosis and long-term quality of life for children suffering from KD complicated by CAAs.

\section{Trial status}

Chinese Clinical Trial Registry, ChiCTR1800019181. Registered on Oct 30, 2018. Currently recruiting, the recruitment start date was Nov 1,2018, and the anticipated recruitment end date is Aug 30, 2021.

\section{Acknowledgments}

We thank the efforts of the patients who participated in our study.

Funding: This study is supported by the following: (I) The Key Project of Capital Clinical Characteristic Application Research (grant number Z181100001718189) (II) The Beijing Hospital Administration "Peak Climbing" Talents Development Program (grant number DFL20181301). 


\section{Footnote}

Reporting Checklist: The authors have completed the SPIRIT reporting checklist. Available at http://dx.doi.org/10.21037/ tp-21-74

Peer Review File: Available at http://dx.doi.org/10.21037/ tp-21-74

Conflicts of Interest: All authors have completed the ICMJE uniform disclosure form (available at http://dx.doi. org/10.21037/tp-21-74). The authors have no conflicts of interest to declare.

Ethical Statement: The authors are accountable for all aspects of the work in ensuring that questions related to the accuracy or integrity of any part of the work are appropriately investigated and resolved. The study will be conducted in accordance with the Declaration of Helsinki (as revised in 2013). The study protocol was approved by the ethics board of Children's Hospital Capital Institute of Pediatrics (NO.: SHERLL2018020) and informed consent will be taken from all the parents or legal guardians.

Open Access Statement: This is an Open Access article distributed in accordance with the Creative Commons Attribution-NonCommercial-NoDerivs 4.0 International License (CC BY-NC-ND 4.0), which permits the noncommercial replication and distribution of the article with the strict proviso that no changes or edits are made and the original work is properly cited (including links to both the formal publication through the relevant DOI and the license). See: https://creativecommons.org/licenses/by-nc-nd/4.0/.

\section{References}

1. Newburger JW, Takahashi M, Burns JC, et al. Kawasaki disease. J Am Coll Cardiol 2016;67:1738-49.

2. Burns JC, Glodé MP. Kawasaki syndrome. Lancet 2004;364:533-44.

3. McCrindle BW, Rowley AH, Newburger JW, et al. American Heart Association Rheumatic Fever, Endocarditis, and Kawasaki Disease Committee of the Council on Cardiovascular Disease in the Young; Council on Cardiovascular and Stroke Nursing; Council on Cardiovascular Surgery and Anesthesia; and Council on Epidemiology and Prevention. Diagnosis, treatment, and long-term management of Kawasaki disease: a scientific statement for health professionals from the American Heart Association. Circulation 2017;135:e927-99.

4. Li JS, Yow E, Berezny KY, et al. Dosing of clopidogrel for platelet inhibition in infants and young children: primary results of the Platelet Inhibition in Children On cLOpidogrel (PICOLO) trial. Circulation 2008;117:553-9.

5. Kato H, Sugimura T, Akagi T, et al. Long-term consequences of Kawasaki disease. A 10- to 21-year followup study of 594 patients. Circulation 1996;94:1379-85.

6. Friedman KG, Gauvreau K, Hamaoka-Okamoto A, et al. Coronary Artery Aneurysms in Kawasaki Disease: Risk Factors for Progressive Disease and Adverse Cardiac Events in the US Population. J Am Heart Assoc 2016;5:e03289.

7. Tsuda E, Kamiya T, Kimura K, et al. Coronary artery dilatation exceeding $4.0 \mathrm{~mm}$ during acute Kawasaki disease predicts a high probability of subsequent late intimamedial thickening. Pediatr Cardiol 2002;23:9-14.

8. Bang JS, Kim GB, Kwon BS, et al. Long-Term Prognosis for Patients with Kawasaki Disease Complicated by Large Coronary Aneurysm (diameter $\geq 6 \mathrm{~mm}$ ). Korean Circ J 2017;47:516-22.

9. Suda K, Iemura M, Nishiono $\mathrm{H}$, et al. Long-term prognosis of patients with Kawasaki disease complicated by giant coronary aneurysms: a single-institution experience. Circulation 2011;123:1836-42.

10. Patrono C, Bachmann F, Baigent C, et al. Expert consensus document on the use of antiplatelet agents. The task force on the use of antiplatelet agents in patients with atherosclerotic cardiovascular disease of the European society of cardiology. Eur Heart J 2004;25:166-81.

11. Israels SJ, Michelson AD. Antiplatelet therapy in children. Thromb Res 2006;118: 75-83.

12. Patel AS, Bruce M, Harrington W, et al. Coronary artery stenosis risk and time course in Kawasaki disease patients: experience at a US tertiary pediatric center. Open Heart 2015;2:e00206.

13. Frelinger AL 3rd, Furman MI, Linden MD, et al. Residual arachidonic acid-induced platelet activation via an adphosphate-dependent but cyclooxygenase- 1 and cyclooxygenase-2 independent pathway: a 700-patient study of aspirin resistance. Circulation 2006;113: 2888-96.

14. Labarthe B, Théroux P, Angioï M, et al. Matching the evaluation of the clinical efficacy of clopidogrel to platelet function tests relevant to the biological properties of the drug. J Am Coll Cardiol 2005;46:638-45.

15. Yusuf S, Zhao F, Mehta SR, et al. Effects of clopidogrel 
in addition to aspirin in patients with acute coronary syndroms without ST-segment elevation. N Engl J Med 2001;345:494-502.

16. Diener HC, Bogousslavsky J, Brass LM, et al. Aspirin and clopidogrel compared with clopidogrel alone after recent ischaemic stroke or transient ischaemic attack in high-risk patients (MATCH): randomised, double-blind, placebocontrolled trial. Lancet 2004;364:331-7.

17. Low T, Jegatheeswaran A, Devlin PJ, et al. Bleeding risk associated with combination thromboprophylaxis therapy is low for patients with coronary artery aneurysms after Kawasaki disease. Int J Cardiol 2020;321:6-11.

Cite this article as: Li D, Chen X, Li X, Yuan Y, Jin H, Liu G, Zhang H, Xie G. Effectiveness and safety of dual antiplatelet therapy in coronary aneurysms caused by Kawasaki disease in children: study protocol for a multicenter randomized clinical trial. Transl Pediatr 2021;10(7):19141923. doi: 10.21037/tp-21-74
18. Su Q, Li J, Tang Z, et al. Association of CYP2C19 Polymorphism with Clopidogrel Resistance in Patients with Acute Coronary Syndrome in China. Med Sci Monit 2019;25:7138-48.

19. Daniels LB, Tjajadi MS, Walford HH, et al. Prevalence of Kawasaki disease in young adults with suspected myocardial ischemia. Circulation 2012;125:2447-53.

20. Huang SK, Lin MT, Chen HC, et al. Epidemiology of Kawasaki disease: prevalence from national database and future trends projection by system dynamics modeling. J Pediatr 2013;163:126-31. 